\title{
Health State Utilities for Economic Evaluation of Bariatric Surgery: A Comprehensive Systematic Review and Meta-Analysis
}

\begin{abstract}
Health-state utilities (HSUs) are health economic metrics that capture and assess healthrelated quality of life (HRQoL). They are essential in health-economic evaluations when calculating quality-adjusted life years. We investigated published studies reporting bariatric surgery-related HSUs elicited through direct or indirect (multi-attribute utility instrument [MAUI]) patient-reported methods (PROSPERO registration number: CRD42019131725). Mean HSUs for different time points and HSU changes over time (where feasible) were meta-analysed using random-effects models. Of the 950 potentially relevant identified studies, n=28 (2004-2018) qualified for data extraction, with $n=85$ unique HSUs elicited mainly from the EQ-5D (88\%). Most (75\%) studies were published after 2013. The follow-up duration varied between studies and was often limited to 12 months. The pooled mean HSU was 0.72 $(0.67-0.76)$ at baseline/pre-surgery $(\mathrm{n}=18)$ and $0.84(0.79-0.89)$ one-year post-surgery ( $n=11)$, indicating a $0.11(0.09-0.14)$ utility unit increment. EQ-5D showed the similar results. This positive difference can be partially explained by BMI and/or co-morbidities status improvement. This study provides a valuable summary of HSUs to future bariatric surgeryrelated cost-utility models. However, more well-designed higher-quality bariatric-related HSU studies are expected for future reviews to improve the available evidence. We suggest that researchers select a MAUI that is preferentially sensitive to the study population.
\end{abstract}

This is the author manuscript accepted for publication and has undergone full peer review but has not been through the copyediting, typesetting, pagination and proofreading process, which may lead to differences between this version and the Version of Record. Please cite this article as doi: 10.1111/obr.13028

This article is protected by copyright. All rights reserved. 
Abbreviations: 15D, 15-dimensional questionnaire; \% EBMIL, percentage excess BMI loss; AGB, adjustable gastric banding; AQoL-8D, Assessment of Quality of Life 8-dimension; ASMBS, American Society for Metabolic and Bariatric Surgery; BMI, body mass index; CHEERS, Consolidated Health Economic Evaluation Reporting Standards; CI, confidence interval; CUA, cost-utility analysis; CVD, cardiovascular disease; EQ-5D, EuroQoL fivedimensions scale; MA-II, Moorehead-Ardelt II questionnaire; MAUI, multi-attribute utility instrument; HRQoL, health-related quality of life; HSUs, Health-state utilities; HUI, Health Utilities Index; PRISMA, Preferred Reporting Items for Systematic Reviews and MetaAnalyses; QALY, quality-adjusted life year; QWB, Quality of Well-Being Scale; RYGB, Roux-en-Y gastric bypass; SD, standard deviation; SF-6D, Short Form-6-dimension; SG, sleeve gastrectomy; T2DM, type 2 diabetes mellitus; TTO, time trade-off; WMD, weighted mean difference.

\section{INTRODUCTION}

\subsection{Obesity and bariatric surgery}

Obesity (defined as body mass index $[\mathrm{BMI}] \geq 30 \mathrm{~kg} / \mathrm{m}^{2}$ ) is epidemic worldwide. ${ }^{1}$ Based on the World Health Organization's most recent data, $13 \%$ of adults worldwide were obese. ${ }^{2}$ Moreover, the proportion of people with severe (BMI $\geq 35 \mathrm{~kg} / \mathrm{m}^{2}$ ) and morbid (BMI $\geq 40$ $\mathrm{kg} / \mathrm{m}^{2}$ ) obesity is rising at a faster rate than obesity. ${ }^{3-5}$ Obesity poses significant health risks (including physical and psychosocial co-morbidities and premature death) to the lives of affected patients, particularly for people who are morbidly obese. ${ }^{6,7}$ Beyond health risks, obesity has also been consistently shown to negatively impact health-related quality of life (HRQoL). ${ }^{8}$ Moreover, obesity imposes substantial economic burden from individual, health 
payer and societal perspectives for developed and developing countries due to, for example, direct healthcare costs and numerous indirect costs including productivity losses. ${ }^{9,10}$

Efficacious interventions to prevent or treat obesity are urgently needed to minimise the adverse HRQoL and direct and indirect cost consequences associated with this condition. An efficient healthcare system needs to offer cost-effective treatments for all diseases including obesity because of healthcare resource scarcity. ${ }^{11}$ Based on the analysis of substantial primary data, we previously found that bariatric (weight loss, metabolic) surgery is a cost-saving treatment for patients with obesity over the lifetime. ${ }^{12,13}$ Besides, bariatric surgery has been reported to be the only clinically effective and sustainable approach to severe and resistant obesity. ${ }^{14,15}$ In addition to actual weight loss and the possible resolution of obesity-related comorbidities following surgery, another widely recognised important outcome measure is the HRQoL improvement.

\subsection{Health-state utilities (HSUs) and associated measures}

Cost-utility analysis (CUA), a type of cost-effectiveness analysis, has become increasingly popular in assisting the decision-making process in several countries, particularly for government reimbursement decisions. ${ }^{16-18}$ In CUA, the cost effectiveness (or even cost savings) of an intervention is expressed in terms of the incremental cost per quality-adjusted life year (QALY), where the QALY combines increased life expectancy (quantity of life) and improvements in health status (quality of life). ${ }^{19} \mathrm{QALY}$ is a potentially useful measure of outcome for decision-making processes and enable direct comparisons to be made between treatment alternatives across various conditions. In order to generate QALYs, preference- 
based HRQoL weights (also known as HSUs) are combined with the length of time spent in the health states of interest. ${ }^{20}$

HSUs are scored between ' 0 ' representing death and ' 1 ' representing perfect health, and negative values represent health states deemed to be worse than death. ${ }^{21}$ Methods for measuring HSUs for economic evaluation included direct and indirect scaling methods. ${ }^{22}$ Direct measurement asks the person to value directly their own health under conditions of uncertainty by using a valuation technique such as time trade-off (TTO) or standard gamble methods. ${ }^{23}$ Indirect measurement involves the use of multi-attribute utility instruments (MAUIs) such as the European EuroQoL five-dimensions scale (EQ-5D; http://www.euroqol.org), the Canadian Health Utilities Index (HUI; http://www.healthutilities.com), the UK Short Form-6-dimension (SF-6D; http://www.shef.ac.uk/scharr/sections/heds/mvh/sf-6d), the Finnish 15-dimensional questionnaire (15D; http://www.15d-instrument.net/15d), the US Quality of Well-Being Scale (QWB; https://eprovide.mapi-trust.org/instruments/quality-of-well-being-scale) and the Australian Assessment of Quality of Life 8-dimension (AQoL-8D; http://www.aqol.com.au), where patients complete the instrument's array of questions on their current health state and these responses are scored using a value set obtained from the general population. ${ }^{24,25}$ With a burgeoning literature of HSUs, which values should be used as inputs in a model is becoming a challenging task. As these MAUIs are far from identical in terms of their descriptive system and covered health states, the choice of MAUI may affect the estimated HSUs, and in turn the CUA outcomes. ${ }^{26}$ Supplement 1 provides a detailed comparison of the characteristics of these instruments. QWB is the first reported MAUI which was released in 1976 in the US, and the 
AQoL-8D is the most recently developed MAUI targeting both physical and psychosocial health dimensions. The EQ-5D dominates the economic evaluation literature (up to 63\% of all MAUIs), as expected from the recommendations of the National Institute for Health and Care Excellence (NICE) guidelines to use the EQ-5D as the preferred measure of HRQoL in adults. $^{27}$

\subsection{Bariatric surgery-related HRQoL and research gaps}

A number of structured or systematic reviews have shown that the HRQoL of patients suffering from obesity improved after surgery. ${ }^{28-33}$ However, these studies are mainly comprised of disease-specific (e.g., Moorehead-Ardelt II questionnaire [MA-II]) or nonpreference-based (e.g., Short Form [SF]-36) data that are unsuitable for cost-utility comparisons because they do not generate a utility value. An emerging literature has shown that HSUs have been used as a metric for quantifying HRQoL improvements regarding bariatric surgery, ${ }^{34-61}$ nevertheless, meta-analyses of bariatric surgery-related HSUs have not been conducted to date. This is surprising, especially given the large number of routinely conducted economic evaluations of bariatric surgeries. ${ }^{62-68}$ Consequently, these modelling studies have not been able to benefit from the meta-analytical HSU estimates, and most rely on the same source of HSUs, with variation in HSU assumptions across models. ${ }^{65,69-71}$ HSUs from single study may not always be a reliable indicator of underlying HRQoL, particularly, where HSUs is not the main focus of the research. Meta-analyses, on the other hand, have the advantage of combining all published HSUs for a given population to maximize the generalizability and representativeness of the estimates used in any economic model, as well as providing insight into the factors that influence HRQoL. ${ }^{21}$ 


\subsection{Objectives of our systematic review}

We acknowledge the current debate regarding HSUs generated by differing MAUIs predominantly because of their descriptive systems, nevertheless, no previous studies have comprehensively investigated and meta-analysed HSU evidence regarding bariatric surgery. Therefore, to address this important research gap, this comprehensive systematic review and meta-analysis aims to generate a database of HSUs that could be used to populate future model-based cost-utility analyses of bariatric surgery procedures. The study as such has two main aims:

1) to undertake a systematic overview of published studies reporting HSU-based HRQoL in patients who underwent bariatric surgery procedures; and

2) to obtain bariatric surgery-related pooled HSUs (from baseline [pre-surgery] to various post-surgery time points) and HSU changes over time (where feasible) that can be used to adjust life expectancy in future CUA.

\section{METHODS}

The protocol of this systematic review was registered on 17 May 2019 at PROSPERO (registration number: CRD42019131725; https://www.crd.york.ac.uk/Prospero/). The search strategy, data screening, extracting and synthesising were followed by the protocol.

\subsection{Validated guidelines}

This systematic review was performed in accordance with the Preferred Reporting Items for Systematic Reviews and Meta-Analyses (PRISMA) statement ${ }^{72}$ and the Campbell and Cochrane Economics Methods Group guidelines. ${ }^{73}$ 


\subsection{Literature search}

Based on previous recommendations ${ }^{74-76}$ and systematic reviews ${ }^{77,78}$ in the field, the literature search was conducted from inception until September 2019 in three biomedical databases (PubMed, EMBASE via OVID and Scopus) and three economic databases (American Economic Association [EconLit], the Cost-Effectiveness Analysis [CEA] Registry, and the Centre for Reviews and Dissemination [CRD], which includes the Database of Abstracts of Reviews of Effects [DARE], Health Technology Assessment Database [HTA] and National Health Service Economic Evaluation Database [NHS EED]).

To ensure literature search saturation, a keyword search of Google Scholar, hand searches and citation tracking of all selected articles and relevant reviews were performed.

The search strategies were developed in consultation with a research librarian at the University of Tasmania. Since Medical Subject Headings (MeSH) terms provide little coverage of HSUs, we identified relevant free-text terms by referring to the published recommendations ${ }^{74,76,79}$ and recent analogous systematic reviews ${ }^{77,80}$. The search strategy combined terms for direct and indirect methods of HSUs elicitations with terms for bariatric surgery. We initially kept the scope and identification of the evidence for HSUs broad. Supplement 2 outlines the initial search strategy for EMBASE via OVID database, which was also adapted for both biomedical and economic databases. Economic filters were considered when searching for evidence on generalist databases, such as PubMed. A simplified search was undertaken without using economic filters for evidence on economics databases such as EconLit. 
Search results were exported to the Covidence online program (Veritas Health Innovation, Melbourne, Australia; https://www.covidence.org), and duplicate articles were removed before screening. Screening of eligible studies was conducted in three steps: first, titles and abstracts were screened by two co-authors (QX and JC) for evidence relevant to HSUs related to bariatric surgery; second, the two co-authors independently assessed the full-text of the remaining studies based on the inclusion criteria of qualitative analysis; third, shortlisted articles with requisite HSU data were further considered for data extraction/synthesis for the meta-analysis. Two additional co-authors (AP and HA) were consulted for a final decision in case of any discrepancies. The number of records identified, retrieved, screened, assessed, included, and excluded in the review, and reasons for exclusions, is summarized in a PRISMA flow diagram (Figure 1). Referencing was managed in EndNote X8.2 (Thomson Reuters).

\subsection{Study eligibility}

Studies were considered eligible if: 1 ) included participants (BMI $\geq 30 \mathrm{~kg} / \mathrm{m}^{2}$ ) undergoing any type of bariatric surgery with assessment of HSUs as primary or secondary patient-reported outcome measures; 2) based on direct (e.g., TTO, standard gamble) or indirect (MAUI based) HSUs elicitation methods; and 3) published as full text (with no time restrictions) in English, German, Chinese, French and Italian languages.

Systematic reviews, meta-analyses, conference abstracts, editorials, letters as well as case reports were excluded. Studies that obtained HSU estimates from previously published literature other than patient-reported outcomes through research or those reporting 
simulation-based utilities were also excluded. If articles referred to the same study population and reported multiple HSUs estimates, the article with the most comprehensive data or the most relevant HSU measures were retained for data syntheses. Where the required HSU data for meta-analytical synthesis were missing, corresponding authors were contacted to improve the comprehensiveness of our study's meta-analyses. If the HSU data could not be provided, the study was excluded from the meta-analysis but still retained in the systematic review.

\subsection{Data extraction}

In order to foster the accuracy of data extraction, a preliminary data collection form was used to extract data from $10 \%$ of studies. Adjustments and improvements were made to the initial form. The first author (QX) used the improved data form to extract data independently, and then the co-author (JC) performed cross-checking. Discrepancies were resolved by consensus, and if agreement was not reached, two additional reviewers (AP and HA) were consulted to reach an agreement. Reviewers were not blinded to information about the authors, author affiliation, and journal name, because this has been shown to be unnecessary. ${ }^{81}$ If HSUs had to be read from a graph, the figures were extracted using the plot digitizing tool WebPlotDigitizer (Version 4.2). ${ }^{82}$

The data extraction was performed according to the requirements of the Cochrane Handbook for Systematic Review of Interventions (version 5.1.0) ${ }^{73}$ and previous guidelines. ${ }^{74,76}$

The following information was extracted from primary studies: first author's name, year of publication, study location, study design of questionnaire assessment (retrospective or prospective), surgery type, comparison (e.g., before versus after, surgical versus non-surgical), 
target population, participants’ sociodemographic characteristics (e.g., female proportion and mean age) at baseline, HSU elicitation method, modes of questionnaire administration (selfor interviewer-administered), types of value set used (local or foreign), sample size, reasons for participant dropout of the study, BMI and obesity-related co-morbidities in each observational time-point, number of follow-ups, follow-up durations (short-term follow-up was defined as from 1 year to 3 year according to the American Society for Metabolic and Bariatric Surgery [ASMBS] outcome reporting standards) ${ }^{83}$, and HSUs in each observational time-point (mean and standard deviation [SD]). Where possible, the individual dimension scores or the proportions of individual dimension responses to each MAUI were extracted to identify the health aspects that were most impacted by bariatric surgery. In addition, the predictors for HSU changes (because of bariatric surgery) were also extracted from the primary studies.

Where HSU data were available only by respondent subgroups based on patient characteristics (e.g., sex), a weighted average of the change score and the score's precision estimate with weights proportional to the subgroup's size were obtained. ${ }^{73}$ When this subgrouping was based on the alternative elicitation methods as described above (i.e., the type of direct-report) or the type of bariatric surgery, then each subgroup was considered independently.

\subsection{Statistical methods for meta-analysis}

We performed a meta-analysis to estimate a single HSU for each time point (i.e., baseline [pre-surgery] and follow-up [post-surgery] time points). Additionally, statistical meta- 
analyses to summarize the weighted mean difference (WMD) in HSU estimates before [baseline/pre-surgery] and after [one-year post-surgery] bariatric surgery were also performed in the form of forest plots.

All statistical analyses and graphing were carried out in STATA (STATA 15.1, StataCorp, College Station, Texas, USA). The command "metan” was used to conduct meta-analysis. ${ }^{84}$ Random-effects models were used because of the variable nature of the source populations for each study to accommodate for likely between-study heterogeneity. ${ }^{85}$

Additional variability in meta-analysis estimates may stem from inter-MAUI differences related to instrument scales, instrument descriptive/classification systems and the sophisticated algorithms that derive the HSUs. Therefore, to examine the nature of these potential sources of heterogeneity, subgroup analyses regarding different elicitation methods were performed. Subgroup analyses for WMD before and after bariatric surgery were only estimated for studies using the EQ-5D instrument, owing to the small number of included studies using other instruments. Additionally, potential heterogeneity based on different surgery types and gender groups was also examined.

Formal quality assessment of primary studies eliciting utility values was not undertaken, given a lack of standard systems or checklists for grading the quality of such studies. ${ }^{74,76}$ Consequently, we examined potential publication and small study bias, visually, using funnel plots (where a symmetrical plot suggested no or little bias) and using Egger's regression asymmetry test (where a $p$-value of $<0.1$ indicated a statistically significant difference). Sensitivity analyses were undertaken to evaluate the stability of the meta-analysis results. 
Individual studies were excluded from all meta-analyses sequentially to gauge the influence of individual studies on the overall results (by using STATA "metaninf” command). ${ }^{86}$ When the remaining pooled rates were not substantially altered, the results of this meta-analysis were deemed stable.

\section{RESULTS}

\subsection{Eligible studies}

Figure 1 presents the process of study selection based on PRISMA methodology. The search strategy yielded 950 citations, with 889 (94\%) citations from biomedical databases, 55 (6\%) from economic databases and six $(<1 \%)$ from other sources. After removing duplicates 676 potentially relevant publications remained for title and abstract screening, with 137 studies included for full-text review. Of these, $n=28$ studies were included in the qualitative synthesis. We subsequently excluded 12 more studies as they either fully or partially relied on the same data sample, ${ }^{34,46,54,58,59,61}$ or did not report requisite HSU data. ${ }^{36,40,48,49,51,56}$ Consequently, only 16 studies were eligible for the meta-analysis. ${ }^{35,37-39,41-45,47,50,52,53,55,57,60}$

\subsection{Study characteristics of the qualitative synthesis $(n=28)$}

Overall, the 28 included studies reported a total of 85 unique HSUs related to bariatric surgery. Supplement 3 provides summary characteristics of the included studies, while all reported HSUs were summarised in Supplement 4. The first study regarding HSUs of bariatric surgery was published in $2004,{ }^{34}$ and most articles ( $\left.n=21 ; 75 \%\right)$ were published after 2013 (Figure 2) which is consistent with the increased popularity of health economic evaluations of bariatric surgery in recent years. Only a small fraction of these studies $(n=5$; 
$18 \%)$ was published in health economics journals, ${ }^{37,41,54,55,59}$ and the vast majority $(n=23$; 82\%) in general medical journals.

Of the included studies, half ( $n=14,50 \%$ ) were conducted in Europe (Finland [ $n=2]$, Spain [n=2], Netherlands [n=2], UK [n=2], Sweden [n=3], France [n=1] and Multicentre within Europe [n=2]), 28\% from North America ( $n=8$ : US [n=4] and Canada [ $n=4]), 11 \%(n=3)$ from Australia, and 11\% from Asia ( $n=3$ : South Korea [n=2] and Israel $[n=1])$. This concurred with the distribution of health economic evaluations of bariatric surgery worldwide. $^{12,13}$ Notably, no study was conducted in developing countries.

Roux-en-Y gastric bypass (RYGB), adjustable gastric banding (AGB) and sleeve gastrectomy (SG) were the three most prevalent procedures, with RYGB and AGB predominantly reported in earlier years, and SG in later years, and also reflecting the technical change of bariatric surgery. ${ }^{13,87,88}$

Study designs included: retrospective studies $(n=8,29 \%)$ and prospective studies $(n=20$, 71\%). Across the twenty-eight identified studies, the baseline sample sizes varied between 23 and 893 (Mean: 194). The dropout was only occurred and was subsequently reported in $n=9$ studies, with n=4 studies also providing the reasons for dropout (Supplement 3).

Whilst most HSU estimates were available for multiple follow-up times (between 2 and 60 months), the follow-up duration varied between studies and was often limited to up-to 12 months (short-term follow-up). Synthesis of HSUs in each observational time point was shown in Section 3.3. 
The mean age in many (23 out of 28, 82\%) included studies ranged between 40 and 50 years. As to sex distribution, all studies except one reported a largely female dominance (average female proportion: $76 \%$ ). Of the 26 studies that provided clear evidence regarding the BMI categories of obesity and associated co-morbidities, $n=17$ (65\%) focused on patients with morbid obesity. The mean BMI at baseline was $45.65(44.13,47.16) \mathrm{kg} / \mathrm{m}^{2}$. On average, the BMI decreased by $13.81 \mathrm{~kg} / \mathrm{m}^{2}$ (95\% CI: 11.02-16.59) one-year post-surgery. Similarly, most studies $(n=17)$ reported the improvements in co-morbidities' status of patients with obesity as a result of surgery, with T2DM and cardiovascular disease (CVD, mainly hypertension in this study) being the main beneficiaries.

In $63 \%$ of cases, HSU estimates for bariatric surgery were based on self-administered surveys, whereas $30 \%$ were based on interview-administered surveys. All HSU estimates were obtained indirectly using MAUIs, and no directly estimated HSUs were identified (Supplement 4). Of the 85 recorded HSUs, $n=75$ (88\%) were measured using the EQ-5D suite of instruments and the associated value set for the particular country (3L: $n=66$; $5 \mathrm{~L}$ : $n=6)$. The remaining HSUs were based on the 15D ( $n=4 ; 5 \%)$, AQoL-8D ( $n=4 ; 5 \%)$, and SF6D ( $n=2 ; 2 \%)$. Most HSU estimates ( $n=64,75 \%)$ were elicited using the local population norms. HSUs were elicited using a single MAUI in most studies: 21 studies used the EQ-5D and two studies the 15D MAUI. In addition, there were five studies that used multiple MAUIs: three used the EQ-5D and AQoL-8D; and two used EQ-5D and SF-6D.

\subsection{Impact of bariatric surgery on HSUs $(n=16)$}


Tables 1 and 2 present the overall as well as subgroup pooled mean HSU estimates at baseline and different follow-up durations. The pooled mean HSU estimate was 0.72 (0.67$0.76), 0.81(0.77-0.85)$ and $0.84(0.79-0.89)$ units at baseline, six-month and one-year postsurgery, respectively (Table 1, Figure 3 [A] and [B]). HSUs captured by SF-6D and AQoL8D were significantly lower than those from the EQ-5D suite of instruments and 15D (Table 1). However, the SF-6D, AQoL-8D and 15D-based HSUs in this comparison were only supported by single studies (Table 1). HSUs were similar among different surgery types (Table 2). Pooled results based on gender difference were not estimated due to insufficient data (Supplement 4).

Overall, the HSU estimate increased a $0.11[0.09,0.14]$ utility unit one-year post-surgery (Figure $3[\mathrm{C}]$ ). Similar results were identified for the EQ-5D suite of instruments. On average, the EQ-5D HSU increased by $0.13(0.09,0.17)$ utility units one-year after bariatric surgery (Figure $3[\mathrm{C}]$ ).

As shown in two studies, ${ }^{44,47}$ patients' HSU improved more for the surgical group than the non-surgical group (Supplement 4), however, the combined data could not be further pooled and then analysed due to varying follow-up durations.

\subsection{Impact of bariatric surgery on individual health dimensions}

Five studies reported the proportion of individual dimension responses to the EQ-5D suite of instruments. As shown in Table 3, patients presented at baseline with more HRQoL impacts for the health dimensions of mobility, usual activities and pain/discomfort, and not a large 
impact on self-care. Improvements in the distribution of responses were recorded one-year post-surgery in four out of the five EQ-5D dimensions (i.e., mobility, usual activities, pain/discomfort and anxiety/depression), especially for mobility and usual activities.

Scores for the individual dimensions of the 15D (baseline vs. one-year) were available in one study. ${ }^{39}$ At baseline, larger HRQoL impacts were reported in respiratory, sexuality and usual acts, and improvements were also recorded in these dimensions one-year after surgery (Supplement $5[\mathrm{~A}])$.

The AQoL-8D's individual dimensional scores were available in only one study for baseline versus one-year comparison. ${ }^{55}$ At baseline, HRQoL impacts were larger in the psychosocial dimensions of health (e.g., mental health and happiness) compared to those in the physical dimensions of health (e.g., independent living and pain). The improvements were recorded in all dimensions especially for psychosocial dimensions (Supplement 5 [B]).

\subsection{Predictors of HSU changes due to bariatric surgery}

As shown in Supplement 6, eleven studies investigated the potential predictors of change in HSUs before and after bariatric surgery. The studies were informed by various statistical methods including correlations analysis and multiple regression models. All but one study investigated the relationship of weight/BMI reduction with change in HSUs due to bariatric surgery, with six recording a significant association. Baseline utility was reported as a significant predictor in one study only. No other significant predictors of HSUs change were identified in these studies. 


\subsection{Publication bias and sensitivity analysis}

There was no evidence of publication bias based on Egger's test for HSU at one-year postsurgery $(z=-1.36, P=0.18)$ and HSU changes before versus after one-year surgery $(z=-0.37$, $P=0.71)$. Significant publication bias was found in baseline HSUs $(z=-2.14, P=0.03)$. The trim-and-fill analysis was conducted, but no studies were imputed. After removing one study (the orange marker in Supplement 7 [A]) with extreme HSU data, publication bias was no longer evident $(P=0.185)$ and the mean baseline HSU stabilized $(0.727[0.681,0.773])$. Funnel plots are shown in Supplement 7. Test of influence of an individual study on the overall meta-analysis estimate did not show significant outliers.

\section{DISCUSSION}

Our comprehensive study is the first to systematically summarise and meta-analyse HSUs regarding bariatric surgery treatment for obesity. Our research substantially overcomes the challenges associated with systematically selecting utility data for economic evaluations of bariatric surgery. The meta-analytical estimates that we have generated can be used as inputs to future bariatric surgery-related cost-utility models. We found that HSU estimates before and after bariatric surgery were largely elicited through the EQ-5D MAUIs. Limited studies used the SF-6D, AQoL-8D and 15D MAUIs, and no study used direct methods. A significant increase in mean HSUs was observed after bariatric surgery, particularly within the first postoperative year. We also found that the reporting of HSUs for bariatric surgery reflected the surgical technical change of bariatric surgery globally, ${ }^{13}$ whilst more reporting is called for in proportion to the large number of bariatric surgery-related models. 
All studies included in our comprehensive systematic review supported bariatric surgery as an effective option in improving HRQoL for people with obesity. We found a mean HSU difference of +0.11 units between the baseline and one-year after bariatric surgery $(+0.13$ units for EQ-5D), and this difference exceeds the minimal clinically important difference for all the MAUIs reported in previous studies (from +0.04 units [EQ-5D] to +0.08 units [AQoL8D]). ${ }^{54,55,89-91}$ In conjunction with this positive HSU difference (from baseline to one-year after bariatric surgery), our study also observed a substantial decrease in the mean BMI. Six out of ten studies reported an inverse relationship between weight/BMI and HSU postsurgery. The findings are in line with a systematic review of reviews that found a significant negative association between obesity and HRQoL, and that the relationship between weight loss and improved HRQoL was consistently demonstrated after bariatric surgery. ${ }^{92}$ Besides, metabolic co-morbidities are reported to be associated with impaired HRQoL among patients who suffered from obesity, and the improvement of these conditions could improve patients' physical and psychosocial quality of life. ${ }^{93}$ Accompanied with the reported weight loss, our study also observed an improvement of co-morbidities' status (e.g., T2DM and CVD) in most of the primary studies. ${ }^{34,38,42,43,46}$ However, most studies did not independently consider the impact of co-morbidities on HSUs. Future studies should examine the impact of changes in co-morbidities status as a result of bariatric surgery on the HSU/HRQoL for patients with obesity. $^{94}$

According to the newest population norms for the EQ-5D-3L for 20 countries, HSUs for the general populations (with healthy body weight) ranged from 0.74 to 0.95 . $^{95}$ Whilst for patients with obesity these values ranged from 0.69 to 0.76 as reported in a double-blind RCT 
study using a TTO score. ${ }^{96}$ In our present study, similar HSU result was identified for people with obesity before undergoing surgery (0.72 [0.67-0.76]); however, an extraordinary higher HSU of 0.84 was reported in a longitudinal survey for waitlist patients through 15D MAUI. ${ }^{39,97}$ Considering the baseline characteristics of these patients (waitlisted, morbidly obese, $83 \%$ comorbidities affected) $)^{39,97}$, we suggest that the key reason for the higher HSU could be due to the relatively young age group (42.1 \pm 10.6 years) considered in this study; otherwise, the use of the 15D MAUI for a waitlisted population's HSUs needs further investigation because HSUs from the other four included studies that investigated patients waiting for bariatric surgery ranged from 0.51 to 0.70 (three from EQ-5D and one from AQoL-8D). ${ }^{42,47,54,55}$

As confirmed by previous studies, heterogeneity among studies may stem from various sources including the differences between various MAUIs due to their different instrument scales, descriptive systems and utility formula. ${ }^{26}$ The choice of the utility assessment method can have a considerable effect on the predicted HSUs and, hence, on the outcome of economic evaluation. ${ }^{98,99}$ For instance, in the present meta-analysis, we found that HSUs captured by the SF-6D and AQoL-8D were significantly lower than those from the EQ-5D and 15D (in particular) (Table 1). Our results should however be interpreted with some caution as being derived from a subgroup meta-analysis based on a limited number of studies (i.e., $\mathrm{n}=2$ for $\mathrm{AQoL}-8 \mathrm{D},{ }^{54,55} \mathrm{n}=1$ for $\mathrm{SF}-6 \mathrm{D}^{37}$ and $\mathrm{n}=2$ for $15 \mathrm{D}^{34,39}$ ).

Whilst the EQ-5D was the most commonly used MAUI to estimate HSUs, there is a debate as to whether the EQ-5D’s descriptive system can capture and assess all the relevant domains of health for people who are waiting for and subsequently undergo bariatric surgery. Our study’s 
findings suggest that the AQoL-8D preferentially captured the psychosocial domains of health compared to the EQ-5D. Moreover, our pooled findings are important because a recent publication in the Journal of the American Medical Association emphasised the importance of measuring the mental health impact of bariatric surgery. ${ }^{100}$ We suggest that this paper's emphasis on mental health directly translates to the notion of choosing a MAUI that is preferentially sensitive to the relevant domains of health for the particular study population. We note that the AQoL-8D MAUI was specifically developed to achieve an increased sensitivity for capturing and assessing the complex physical and psychosocial HRQoL health states that are relatively neglected in other MAUIs, particularly for people with complex and chronic disease states. ${ }^{101,102}$ However, as identified by our present study, only two HSU studies using the AQoL-8D MAUI longitudinally have been published to date. We therefore hope that the use of AQoL-8D or other MAUIs that are sensitive to the relevant domains of health for people with bariatric surgery, may provide more credible HSUs.

In addition to the elicitation method, other potential sources of variation in reported HSU values may include patients’ socio-demographic characteristics (e.g., age, sex or initial BMI), surgery type, follow-up durations, mode of administration and co-morbidities status. However, these may not have a large impact on our meta-analyses results as patient-level HSUs were only considered in our study, and patients' socio-demographic characteristics in our study (i.e., sex proportion, mean age and mean BMI) were generally comparable across included studies (Supplement 8). Through additional subgroup meta-analysis (Table 2), we further established that surgery type did not contribute to this variation. Meta-regression was not possible for all variables due to the insufficient number of included studies. Future studies 
are recommended to investigate the magnitude of effect and relative contribution of each of the pertinent impact factors on HSUs of people who receive bariatric surgery.

Our study has unique strengths. This is the first study to systematically assess and summarise HSUs related to bariatric surgery, which provides a standard set of HSUs that could be used in health economic assessments of bariatric surgery procedures. An advantage of undertaking a meta-analysis is that it provides both an average value as well as extreme values that could be used in a sensitivity analysis. Providing a range of values alongside the summary HSUs is particularly important as there is considerable heterogeneity in the HSUs between bariatric surgery studies. Likewise, a further strength of our study is the broad range of study populations conducted in, for example, different countries and ethnicities, maximising the generalizability and representativeness of the HSU estimates used in bariatric surgery-related economic models.

This systematic review and meta-analyses also has limitations. First, our meta-analytical HSU estimates predominantly relied on the EQ-5D MAUIs with limited coverage of psychosocial health aspects, and potential ceiling and floor effects. Second, HSU follow-up times were generally limited to short term (up to one year), hence, our study failed to capture longer-term changes in HSUs as a result of bariatric surgery. The long-term data from this study should be interpreted with some caution when using in the models. Third, HSU differences between surgical and non-surgical groups were not available in current metaanalysis due to the lack of relevant data. Fourth, we have used mean with SD in data syntheses. The distribution of the HSU values was not reported in most of the primary studies, sometimes these values can be negatively skewed which may bias the pooled HSU estimates 
downwards. Fifth, recall biases could not be neglected from few studies due to the observational nature. Finally, quality appraisal of the included studies was not undertaken in the current study, given a lack of standard systems or checklists for grading the quality of HSU studies; nevertheless, our study is the first gold-standard systematic review and metaanalysis that provides a baseline of studies to date.

Summary of recommendations for future HSU analyses:

- A preferentially sensitive MAUI should be selected that appropriately captures and measures both the complex and interdependent physical and psychosocial health needs of the bariatric surgery study population;

- Before versus after comparisons, to some extent, underestimated the impairment of obesity on HRQoL due to the fact that this study design does not consider the effect of follow-up on patients with obesity who do not receive bariatric surgery. To avoid it, future studies should consider simultaneous control group to balance the time effect among patients with and without surgery;

- Studies with longer time horizons are needed to assess the long-term effects of bariatric surgery on utility based HRQoL;

- Future studies also need to test and report the distribution of HSU data to improve the reporting quality;

- Future studies that examine the HRQoL impact of changes in obesity-associated comorbidities status (as well as other potential factors such as complications and revisional procedures) as a result of surgery are also needed; 
- More HSU research from developing countries are needed. For example, obesity prevalence is increasing, and obesity surgery is also prevalent in China; however, relative to population health economic evaluation is less reported, and despite the inclusion criteria of various languages including Chinese this study did not capture any HSU estimates from China or other similar countries;

- Guidelines or structured recommendations are urgently needed to support authors and reviewers in assessing their study quality.

\section{CONCLUSIONS}

This study represents the first systematic review and meta-analysis of HSUs regarding bariatric surgery treatment for obesity, demonstrating the relative lack of published HSUbased HRQoL for bariatric surgery. We have seen the increasing trend of health economic evaluations of bariatric surgery since 2013. Meta-analysis results suggest that HSU-based quality of life increased at least within one-year after surgery, and this positive difference can be partially explained by BMI and/or co-morbidities status improvement. The meta-analytical estimates that we have generated can be used as more reliable inputs to future bariatric surgery-related cost-utility models. However, more well-designed higher-quality bariatricrelated HSU studies are expected for future reviews to improve the available evidence, including longer follow-up times. ${ }^{103}$

\section{AUTHORS' CONTRIBUTIONS}

QX conceived the study, collaborated in the design and implementation of the study and wrote the paper. JC conceived the study, collaborated in the design and implementation of the 
study, and critically reviewed and edited the manuscript. HA collaborated in the design and implementation of the study, and critically reviewed and edited the manuscript. BdG collaborated in the design of the study and edited the paper. LS collaborated in the design of the study and edited the paper. PO participated in the data syntheses and critically reviewed and edited the manuscript. AP conceived the study, collaborated in the design of the study, and critically reviewed and edited the paper. All authors read and approved the final paper.

\section{CONFLICTS OF INTEREST}

This systematic review has received no funding. Dr. Lei Si is funded by a NHMRC Early Career Fellowship (GNT1139826).

\section{ACKNOWLEDGEMENTS}

We thank Michaela Venn, a librarian at University of Tasmania, for the assistance in developing search strategy of this review. 


\section{LEGENDS:}

Table 1: Meta-analysis (random effects) results in each observational time points, by elicitation method $(n=16)$;

Table 2: Meta-analysis (random effects) results in each observational time points, by surgery type $(n=16)$;

Table 3: Proportion of individual dimension response to EQ-5D instrument (pre-surgery versus post-surgery);

Figure 1: Flow of studies into the narrative systematic review and meta-analyses, informed by PRISMA guidelines;

Figure 2: Number of studies reporting HSUs of bariatric surgery: (A) from 2004 to 2018; (B) before and after the year of 2013;

Figure 3: Forest plots (random-effects) of HSUs regarding bariatric surgery, by elicitation method: (A) baseline HSUs; (B) HSUs at one-year post-surgery; and (C) HSU changes before versus one-year post-surgery;

Supplement 1: Comparisons of the dimensions and content of multi-attribute utility instruments;

Supplement 2: Search strategy for EMBASE via OVID;

Supplement 3: Characteristics of studies included in systematic review $(n=28)$;

Supplement 4: Details of HSUs reported by included studies; 
Supplement 5: Radar charts comparing individual dimensional score before and after bariatric surgery: (A) 15D and (B) AQoL-8D;

Supplement 6: Predictors for HSU changes before and after bariatric surgery;

Supplement 7: Funnel plots of HSUs regarding bariatric surgery: (A) baseline HSUs; (B) HSUs at one-year post-surgery; and (C) HSU changes before versus one-year post-surgery;

Supplement 8: Univariate meta-regression of patients' socio-demographic characteristics in reported HSUs. 


\section{REFERENCES:}

1. Ng M, Fleming $\mathrm{T}$, Robinson $\mathrm{M}$, et al. Global, regional, and national prevalence of overweight and obesity in children and adults during 1980-2013: a systematic analysis for the Global Burden of Disease Study 2013. Lancet. 2014;384:766-81.

2. World Health Organization (WHO). Obesity and overweight Fact Sheet October 2018. http://www.who.int/en/news-room/fact-sheets/detail/obesity-and-overweight. 16 February 2018.

3. Skinner AC, Skelton JA. Prevalence and trends in obesity and severe obesity among children in the United States, 1999-2012. JAMA pediatrics. 2014;168:561-6.

4. Keating C, Backholer K, Gearon E, et al. Prevalence of class-I, class-II and class-III obesity in Australian adults between 1995 and 2011-12. Obes Res Clin Pract. 2015;9:553-62.

5. Sturm R, Hattori A. Morbid obesity rates continue to rise rapidly in the United States. Int J Obes (Lond). 2013;37:889-91.

6. Wang YC, McPherson K, Marsh T, Gortmaker SL, Brown M. Health and economic burden of the projected obesity trends in the USA and the UK. Lancet. 2011;378:815-25.

7. $\quad$ Haslam DW, James WP. Obesity. Lancet. 2005;366:1197-209.

8. Hopman WM, Berger $C$, Joseph $L$, et al. The association between body mass index and health-related quality of life: data from CaMos, a stratified population study. Qual Life Res. 2007;16:1595-603.

9. Tremmel M, Gerdtham U-G, Nilsson PM, Saha S. Economic Burden of Obesity: A Systematic Literature Review. International journal of environmental research and public health. 2017;14:435.

10. Cawley J, Meyerhoefer C. The medical care costs of obesity: an instrumental variables approach. J Health Econ. 2012;31:219-30.

11. Drummond MF, Sculpher MJ, Claxton K, Stoddart GL, Torrance GW. Methods for the Economic Evaluation of Health Care Programmes, 4th Edition. Oxford University Press; 2015.

12. Campbell JA, Venn A, Neil A, Hensher M, Sharman M, Palmer AJ. Diverse approaches to the health economic evaluation of bariatric surgery: a comprehensive systematic review. Obes Rev. 2016;17:850-94.

13. Xia Q, Campbell JA, Ahmad H, Si L, de Graaff B, Palmer AJ. Bariatric surgery is a cost-saving treatment for obesity-A comprehensive meta-analysis and updated systematic review of health economic evaluations of bariatric surgery. Obes Rev. 2019.

14. Buchwald $\mathrm{H}$, Avidor $\mathrm{Y}, \mathrm{Braunwald} \mathrm{E}$, et al. Bariatric surgery: a systematic review and metaanalysis. Jama. 2004;292:1724-37.

15. Chang SH, Stoll CR, Song J, Varela JE, Eagon CJ, Colditz GA. The effectiveness and risks of bariatric surgery: an updated systematic review and meta-analysis, 2003-2012. JAMA Surg. 2014;149:275-87.

16. Neumann PJ, Greenberg D, Olchanski NV, Stone PW, Rosen AB. Growth and Quality of the Cost-Utility Literature, 1976-2001. Value in Health. 2005;8:3-9.

17. Neumann PJ, Thorat T, Shi J, Saret CJ, Cohen JT. The changing face of the cost-utility literature, 1990-2012. Value Health. 2015;18:271-7.

18. Thorat T, Lin PJ, Neumann PJ. The State of Cost-Utility Analyses in Asia: A Systematic Review. Value Health Reg Issues. 2015;6:7-13.

19. Neumann PJ, Sanders GD. Cost-Effectiveness Analysis 2.0. N Engl J Med. 2017;376:203-5. 
20. Torrance GW. Preferences for health outcomes and cost-utility analysis. Am J Manag Care. 1997;3 Suppl:S8-20.

21. Wolowacz SE, Briggs A, Belozeroff V, et al. Estimating Health-State Utility for Economic Models in Clinical Studies: An ISPOR Good Research Practices Task Force Report. Value Health. 2016;19:704-19.

22. Brazier J, Ara R, Rowen D, Chevrou-Severac H. A Review of Generic Preference-Based Measures for Use in Cost-Effectiveness Models. Pharmacoeconomics. 2017;35:21-31.

23. Torrance GW. Utility approach to measuring health-related quality of life. J Chronic Dis. 1987; 40:593-603.

24. Richardson J, McKie J, E B. Review and Critique of Health Related Multi Attribute Utility Instruments. Centre for Health Economics Monash University Business and Economics Research Paper;2011.

25. Stevens KJ. How Well Do the Generic Multi-attribute Utility Instruments Incorporate Patient and Public Views Into Their Descriptive Systems? Patient. 2016;9:5-13.

26. Richardson J, lezzi A, Khan MA. Why do multi-attribute utility instruments produce different utilities: the relative importance of the descriptive systems, scale and 'micro-utility' effects. Quality of Life Research. 2015;24:2045-53.

27. Richardson J, McKie J, Bariola E. Multi attribute utility instruments and their use. Encyclopedia of health economics. San Diego: Elsevier Science. 2014:pp. 341-57.

28. Magallares A, Schomerus G. Mental and physical health-related quality of life in obese patients before and after bariatric surgery: a meta-analysis. Psychol Health Med. 2015;20:165-76.

29. Tayyem R, Ali A, Atkinson J, Martin CR. Analysis of health-related quality-of-life instruments measuring the impact of bariatric surgery: systematic review of the instruments used and their content validity. Patient. 2011;4:73-87.

30. Coulman KD, Abdelrahman T, Owen-Smith A, Andrews RC, Welbourn R, Blazeby JM. Patientreported outcomes in bariatric surgery: a systematic review of standards of reporting. Obes Rev. 2013;14:707-20.

31. Adil MT, Jain V, Rashid F, Al-Taan O, Whitelaw D, Jambulingam P. Meta-analysis of the effect of bariatric surgery on physical function. Br J Surg. 2018;105:1107-18.

32. Raaijmakers LC, Pouwels S, Thomassen SE, Nienhuijs SW. Quality of life and bariatric surgery: a systematic review of short- and long-term results and comparison with community norms. Eur J Clin Nutr. 2017;71:441-9.

33. Lindekilde N, Gladstone BP, Lubeck $M$, et al. The impact of bariatric surgery on quality of life: a systematic review and meta-analysis. Obes Rev. 2015;16:639-51.

34. Tolonen P, Victorzon M, Makela J. Impact of laparoscopic adjustable gastric banding for morbid obesity on disease-specific and health-related quality of life. Obes Surg. 2004;14:788-95.

35. van Mastrigt GA, van Dielen FM, Severens JL, Voss GB, Greve JW. One-year costeffectiveness of surgical treatment of morbid obesity: vertical banded gastroplasty versus Lap-Band. Obes Surg. 2006;16:75-84.

36. Sanchez-Santos R, Del Barrio MJ, Gonzalez C, et al. Long-term health-related quality of life following gastric bypass: influence of depression. Obes Surg. 2006;16:580-5.

37. Sauerland S, Weiner S, Dolezalova K, et al. Mapping utility scores from a disease-specific quality-of-life measure in bariatric surgery patients. Value Health. 2009;12:364-70. 
38. McEwen LN, Coelho RB, Baumann LM, Bilik D, Nota-Kirby B, Herman WH. The cost, quality of life impact, and cost-utility of bariatric surgery in a managed care population. Obes Surg. 2010;20:919-28.

39. Helmio M, Salminen P, Sintonen H, Ovaska J, Victorzon M. A 5-year prospective quality of life analysis following laparoscopic adjustable gastric banding for morbid obesity. Obes Surg. 2011;21:1585-91.

40. Date RS, Walton SJ, Ryan N, Rahman SN, Henley NC. Is selection bias toward super obese patients in the rationing of metabolic surgery justified?--A pilot study from the United Kingdom. Surg Obes Relat Dis. 2013;9:981-6.

41. Lin VW, Wong ES, Wright A, et al. Association between health-related quality of life and body mass after adjustable gastric banding: a nonlinear approach. Value Health. 2013;16:823-9.

42. Mar J, Karlsson J, Arrospide A, Mar B, Martinez de Aragon G, Martinez-Blazquez C. Two-year changes in generic and obesity-specific quality of life after gastric bypass. Eat Weight Disord. 2013;18:305-10.

43. Ribaric G, Buchwald JN, d'Orsay G, Daoud F. 3-year real-world outcomes with the Swedish adjustable gastric band in France. Obes Surg. 2013;23:184-96.

44. Oh SH, Song HJ, Kwon JW, et al. The improvement of quality of life in patients treated with bariatric surgery in Korea. J Korean Surg Soc. 2013;84:131-9.

45. Halperin F, Ding SA, Simonson DC, et al. Roux-en-Y gastric bypass surgery or lifestyle with intensive medical management in patients with type 2 diabetes: feasibility and 1-year results of a randomized clinical trial. JAMA Surg. 2014;149:716-26.

46. Lee YJ, Song HJ, Heo Y, et al. Validation of the Korean version Moorehead-Ardelt quality of life questionnaire II. Ann Surg Treat Res. 2014;87:265-72.

47. Warkentin LM, Majumdar SR, Johnson JA, et al. Weight loss required by the severely obese to achieve clinically important differences in health-related quality of life: two-year prospective cohort study. BMC Med. 2014;12:175.

48. Ding SA, Simonson DC, Wewalka $M$, et al. Adjustable Gastric Band Surgery or Medical Management in Patients With Type 2 Diabetes: A Randomized Clinical Trial. J Clin Endocrinol Metab. 2015;100:2546-56.

49. Wiklund M, Olsén MF. Physical Function and Health Related Quality of Life Before and 18 Months after Bariatric Surgery. The Open Obesity Journal. 2015;7:12-6.

50. Biter LU, van Buuren MMA, Mannaerts GHH, Apers JA, Dunkelgrun M, Vijgen G. Quality of Life 1 Year After Laparoscopic Sleeve Gastrectomy Versus Laparoscopic Roux-en-Y Gastric Bypass: a Randomized Controlled Trial Focusing on Gastroesophageal Reflux Disease. Obes Surg. 2017;27:2557-65.

51. Tarride JE, Breau R, Sharma AM, et al. The Effect of Bariatric Surgery on Mobility, HealthRelated Quality of Life, Healthcare Resource Utilization, and Employment Status. Obes Surg. 2017;27:349-56.

52. Twells LK, Driscoll S, Gregory DM, Lester K, Fardy JM, Pace D. Morbidity and health-related quality of life of patients accessing laparoscopic sleeve gastrectomy: a single-centre crosssectional study in one province of Canada. BMC Obes. 2017;4:40.

53. Fermont JM, Blazeby JM, Rogers CA, Wordsworth S. The EQ-5D-5L is a valid approach to measure health related quality of life in patients undergoing bariatric surgery. PLoS One. 2017; 12:e0189190. 
54. Campbell JA, Hensher M, Neil A, et al. An Exploratory Study: A Head-to-Head Comparison of the EQ-5D-5L and AQoL-8D for Long-Term Publicly Waitlisted Bariatric Surgery Patients Before and 3 Months After Bariatric Surgery. Pharmacoecon Open. 2018;2:443-58.

55. Campbell JA, Hensher M, Neil A, Venn A, Wilkinson S, Palmer AJ. An Exploratory Study of Long-Term Publicly Waitlisted Bariatric Surgery Patients' Quality of Life Before and 1 Year After Bariatric Surgery, and Considerations for Healthcare Planners. Pharmacoecon Open. 2018;2:63-76.

56. Kessler Y, Olmer L, Raziel A, Goitein D, Dankner R. Associations of dietitian follow-up counselling visits and physical exercise with weight loss one year after sleeve gastrectomy. Eat Weight Disord. 2018.

57. Campbell JA, Palmer AJ, Venn A, Sharman M, Otahal P, Neil A. A Head-to-Head Comparison of the EQ-5D-5L and AQoL-8D Multi-Attribute Utility Instruments in Patients Who Have Previously Undergone Bariatric Surgery. Patient. 2016;9:311-22.

58. Sauerland S, Weiner S, Hausler E, et al. Validity of the Czech, German, Italian, and Spanish version of the Moorehead-Ardelt II questionnaire in patients with morbid obesity. Obes Facts. 2009;2 Suppl 1:57-62.

59. Lester EL, Padwal R, Majumdar SR, Ye F, Birch DW, Klarenbach SW. Association of Preference-Based Health-Related Quality of Life with Weight Loss in Obese Adults. Value Health. 2017;20:694-8.

60. Biorserud C, Olbers T, Staalesen T, Elander A, Olsen MF. Understanding excess skin in postbariatric patients: objective measurements and subjective experiences. Surg Obes Relat Dis. 2016;12:1410-7.

61. Biorserud C, Shams K, Elander A, Fagevik Olsen M. Self-image after bariatric surgery and its relationship to gender, excess skin and health-related quality of life. J Plast Surg Hand Surg. 2018:1-6.

62. Ackroyd R, Mouiel J, Chevallier JM, Daoud F. Cost-effectiveness and budget impact of obesity surgery in patients with type-2 diabetes in three European countries. Obes Surg. 2006;16:1488-503.

63. van Gemert WG, Adang EM, Kop M, Vos G, Greve JW, Soeters PB. A prospective costeffectiveness analysis of vertical banded gastroplasty for the treatment of morbid obesity. Obes Surg. 1999;9:484-91.

64. Campbell J, McGarry LA, Shikora SA, Hale BC, Lee JT, Weinstein MC. Cost-effectiveness of laparoscopic gastric banding and bypass for morbid obesity. Am J Manag Care. 2010;16:e174-87.

65. Song HJ, Kwon JW, Kim YJ, Oh SH, Heo Y, Han SM. Bariatric surgery for the treatment of severely obese patients in South Korea--is it cost effective? Obes Surg. 2013;23:2058-67.

66. Borisenko O, Adam D, Funch-Jensen P, et al. Bariatric Surgery can Lead to Net Cost Savings to Health Care Systems: Results from a Comprehensive European Decision Analytic Model. Obes Surg. 2015;25:1559-68.

67. Faria GR, Preto JR, Costa-Maia J. Gastric bypass is a cost-saving procedure: results from a comprehensive Markov model. Obes Surg. 2013;23:460-6.

68. Lucchese M, Borisenko O, Mantovani LG, et al. Cost-Utility Analysis of Bariatric Surgery in Italy: Results of Decision-Analytic Modelling. Obes Facts. 2017;10:261-72. 
69. James R, Salton RI, Byrnes JM, Scuffham PA. Cost-utility analysis for bariatric surgery compared with usual care for the treatment of obesity in Australia. Surg Obes Relat Dis. 2017;13:2012-20.

70. Alsumali A, Eguale T, Bairdain S, Samnaliev M. Cost-Effectiveness Analysis of Bariatric Surgery for Morbid Obesity. Obes Surg. 2018;28:2203-14.

71. Gulliford MC, Charlton J, Prevost T, et al. Costs and Outcomes of Increasing Access to Bariatric Surgery: Cohort Study and Cost-Effectiveness Analysis Using Electronic Health Records. Value Health. 2017;20:85-92.

72. Moher D, Liberati A, Tetzlaff J, et al. Preferred reporting items for systematic reviews and meta-analyses: the PRISMA statement. PLoS medicine. 2009;6:e1000097.

73. Higgins JPT, Green S (editors). Cochrane Handbook for Systematic Reviews of Interventions Version 5.1.0 [updated March 2011]. The Cochrane Collaboration; 2011, Chapter 15. Available from: http://handbook.cochrane.org.

74. Petrou S, Kwon J, Madan J. A Practical Guide to Conducting a Systematic Review and Metaanalysis of Health State Utility Values. Pharmacoeconomics. 2018;36:1043-61.

75. Arber M, Glanville J, Isojarvi J, et al. Which Databases Should Be Used to Identify Studies for Systematic Reviews of Economic Evaluations? Int J Technol Assess Health Care. 2018:1-8.

76. Papaioannou D, Brazier J, Paisley S. Systematic searching and selection of health state utility values from the literature. Value Health. 2013;16:686-95.

77. Meregaglia M, Cairns J. A systematic literature review of health state utility values in head and neck cancer. Health Qual Life Outcomes. 2017;15:174.

78. Si L, Winzenberg TM, de Graaff B, Palmer AJ. A systematic review and meta-analysis of utility-based quality of life for osteoporosis-related conditions. Osteoporos Int. 2014;25:1987-97.

79. Arber M, Garcia S, Veale T, Edwards M, Shaw A, Glanville JM. Performance of Ovid Medline Search Filters to Identify Health State Utility Studies. Int J Technol Assess Health Care. 2017;33:472-80.

80. Moayeri F, Hsueh YS, Clarke P, Dunt D. Do Model-Based Studies in Chronic Obstructive Pulmonary Disease Measure Correct Values of Utility? A Meta-Analysis. Value Health. 2016;19:363-73.

81. Berlin JA. Does blinding of readers affect the results of meta-analyses? University of Pennsylvania Meta-analysis Blinding Study Group. Lancet. 1997;350:185-6.

82. Drevon D, Fursa SR, Malcolm AL. Intercoder Reliability and Validity of WebPlotDigitizer in Extracting Graphed Data. Behav Modif. 2017;41:323-39.

83. Brethauer SA, Kim J, El Chaar M, et al. Standardized outcomes reporting in metabolic and bariatric surgery. Obes Surg. 2015;25:587-606.

84. Harris RJ, Deeks JJ, Altman DG, Bradburn MJ, Harbord RM, Sterne JAC. Metan: Fixed- and Random-Effects Meta-Analysis. The Stata Journal. 2008;8:3-28.

85. Borenstein M, Hedges LV, Higgins JP, Rothstein HR. A basic introduction to fixed-effect and random-effects models for meta-analysis. Res Synth Methods. 2010;1:97-111.

86. Xia $Q$, Fan $D$, Yang $X$, et al. Progression rate of ankylosing spondylitis in patients with undifferentiated spondyloarthritis: A systematic review and meta-analysis. Medicine (Baltimore). 2017;96:e5960.

87. Angrisani L, Santonicola A, lovino P, Formisano G, Buchwald H, Scopinaro N. Bariatric Surgery Worldwide 2013. Obes Surg. 2015;25:1822-32. 
88. Khorgami Z, Shoar S, Andalib A, Aminian A, Brethauer SA, Schauer PR. Trends in utilization of bariatric surgery, 2010-2014: sleeve gastrectomy dominates. Surg Obes Relat Dis. 2017;13:774-8.

89. Crosby R, Kolotkin R, Williams G. Defining clinically meaningful change in health-related quality of life. Journal of clinical epidemiology. 2003;56:395-407.

90. Hawthorne G, Osborne R. Population norms and meaningful differences for the Assessment of Quality of Life (AQoL) measure. Aust N Z J Public Health. 2005;29:136-42.

91. Walters SJ, Brazier JE. What is the relationship between the minimally important difference and health state utility values? The case of the SF-6D. Health Qual Life Outcomes. 2003;1:4.

92. Kolotkin RL, Andersen JR. A systematic review of reviews: exploring the relationship between obesity, weight loss and health-related quality of life. Clin Obes. 2017;7:273-89.

93. UI-Haq Z, Mackay DF, Fenwick E, Pell JP. Impact of metabolic comorbidity on the association between body mass index and health-related quality of life: a Scotland-wide cross-sectional study of 5,608 participants. BMC Public Health. 2012;12:143.

94. Lung TW, Hayes AJ, Hayen A, Farmer A, Clarke PM. A meta-analysis of health state valuations for people with diabetes: explaining the variation across methods and implications for economic evaluation. Qual Life Res. 2011;20:1669-78.

95. Janssen MF, Szende A, Cabases J, Ramos-Goni JM, Vilagut G, Konig HH. Population norms for the EQ-5D-3L: a cross-country analysis of population surveys for 20 countries. Eur J Health Econ. 2019;20:205-16.

96. Hakim Z, Wolf A, Garrison LP. Estimating the effect of changes in body mass index on health state preferences. PharmacoEconomics. 2002;20:393-404.

97. Victorzon $\mathrm{M}$, Tolonen $\mathrm{P}$, Sintonen $\mathrm{H}$. Health-related quality of life in severely and morbidly obese patients waiting for bariatric surgery in Finland. Scand J Surg. 2010;99:122-6.

98. Conner-Spady B, Suarez-Almazor ME. Variation in the estimation of quality-adjusted lifeyears by different preference-based instruments. Med Care. 2003;41:791-801.

99. Kopec JA, Willison KD. A comparative review of four preference-weighted measures of health-related quality of life. Journal of Clinical Epidemiology. 2003;56:317-25.

100. Dawes AJ, Maggard-Gibbons M, Maher AR, et al. Mental Health Conditions Among Patients Seeking and Undergoing Bariatric Surgery: A Meta-analysis. Jama. 2016;315:150-63.

101. Ahmad H, Taylor BV, van der Mei I, et al. The impact of multiple sclerosis severity on health state utility values: Evidence from Australia. Mult Scler. 2017;23:1157-66.

102. Ahmad H, Palmer AJ, Campbell JA, van der Mei I, Taylor BVM. Health economic impact of Multiple Sclerosis in Australia in 2017. An analysis of MS Research Australia's platform - the Australian MS Longitudinal Study (AMSLS). 22 AUG 2018.

103. Hawthorne G, Richardson J. Measuring the value of program outcomes: a review of multiattribute utility measures. Expert Rev Pharmacoecon Outcomes Res. 2001;1:215-28. 


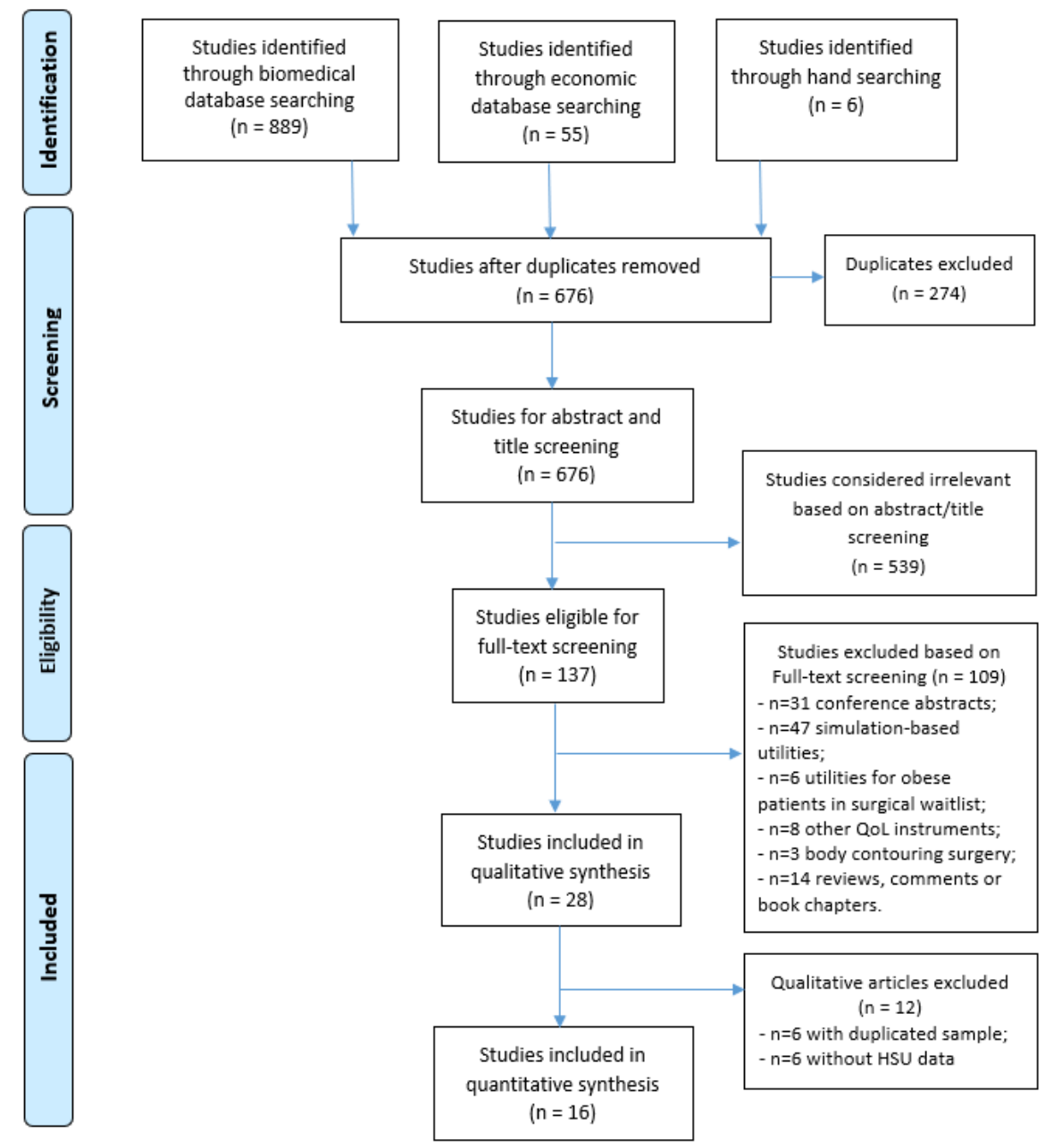

OBR_13028_F1.tif 
(A)

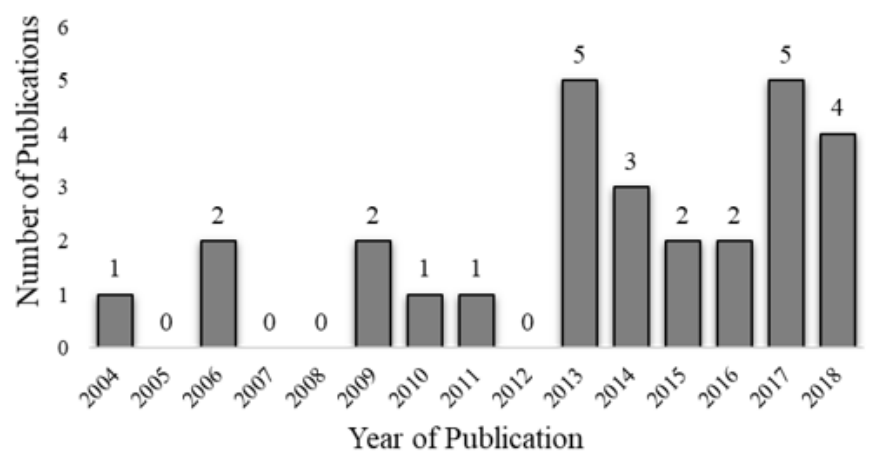

(B)

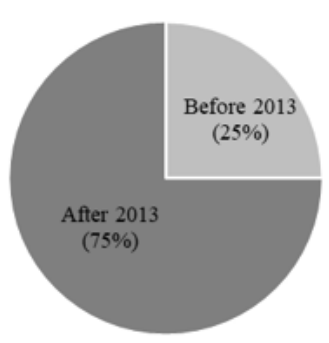

$=$ Before 2013 = After 2013

OBR_13028_F2.tif

This article is protected by copyright. All rights reserved. 

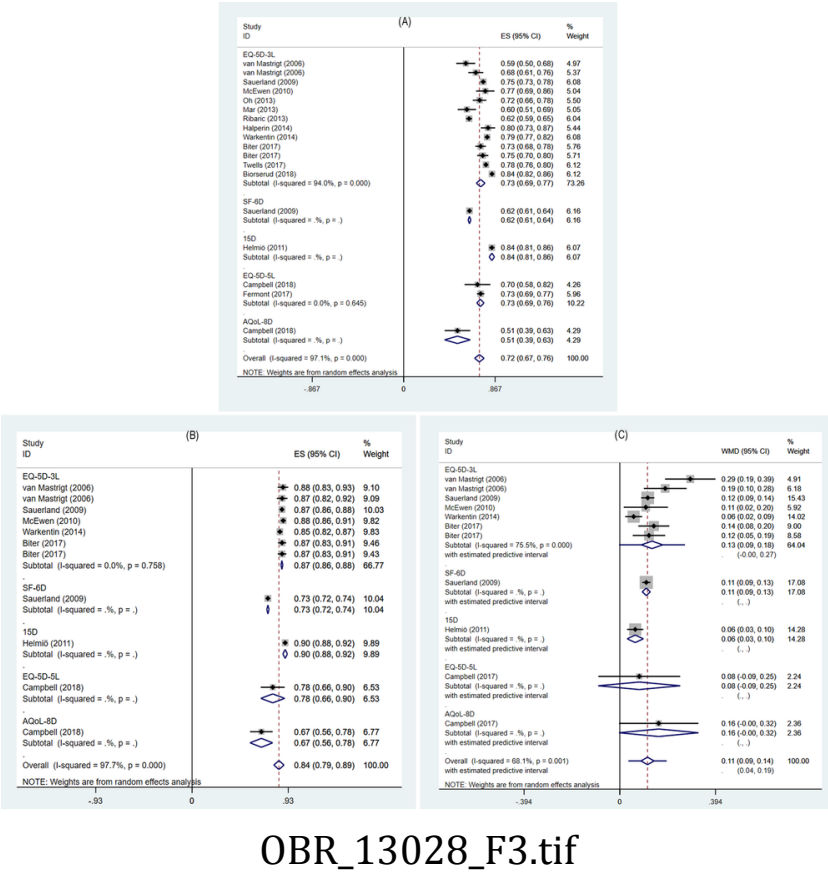

This article is protected by copyright. All rights reserved. 


\section{University Library}

\section{- M M I N E R VA \\ A gateway to Melbourne's research publications}

Minerva Access is the Institutional Repository of The University of Melbourne

Author/s:

Xia, Q;Campbell, JA;Ahmad, H;Si, L;de Graaff, B;Otahal, P;Palmer, AJ

Title:

Health state utilities for economic evaluation of bariatric surgery: A comprehensive systematic review and meta-analysis

Date:

2020-06-04

Citation:

Xia, Q., Campbell, J. A., Ahmad, H., Si, L., de Graaff, B., Otahal, P. \& Palmer, A. J. (2020). Health state utilities for economic evaluation of bariatric surgery: A comprehensive systematic review and meta-analysis. OBESITY REVIEWS, 21 (8), https://doi.org/10.1111/ obr.13028.

Persistent Link:

http://hdl.handle.net/11343/275865 